\title{
Il corpo come linguaggio: tra teorie, pratica e retorica (età martiriale e postmartiriale)
}

\author{
Teresa SARDELLA \\ Università di Catania \\ sardella@unict.it
}

\section{IL LINGUAGGIO DEL CORPO E LA RETORICA DELLA PENITENZA PUBBLICA}

La retorica -l'arte retorica-, quale più antica scienza del linguaggio, insieme alla grammatica, coinvolge saperi oggi distinti e specifici, come filosofia, politica e dialettica. Il suo valore rinvia all'arte del dire e del parlare, del persuadere mediante i discorsi. Inoltre, essa bada a trattenere nel termine arte il tradizionale significato di insieme di tecniche pratiche. Nei valori concreti della comunicazione, contestualizzata in situazioni pratiche di pubblica utilità, fin dalle origini, fu concentrato lo studio formale dei metodi per trattare un linguaggio volto a una comunicazione che avesse le specifiche finalità del coinvolgere e del persuadere.

Con Cicerone entrarono in modo strutturale, e come parte integrante dell'ars retorica, il corpo e, più in particolare, le sue potenzialità comunicative funzionali all'uso del potere ${ }^{1}$ : il corpo come linguaggio, la cui semeiotica era coadiuvante del discorso retorico. Esso entrava, nello specifico, nella organizzazione ad arte della comunicazione gestuale funzionalizzata, con coscienza e consapevolezza, per sostenere un messaggio intellettuale. Ma, si trattava, per l'appunto, della costruzione all'interno di un sistema di comunicazione che intendeva avvalersi coscientemente e coerentemente anche del corpo e dove il messaggio, attraverso una tecnica insegnata, procedeva dall'alto verso il basso ${ }^{2}$. Questa prospettiva è profondamente diversa da quella che è con-

\footnotetext{
${ }^{1}$ Il rapporto tra corpo e potere è leggibile secondo molte chiavi di lettura: oltre che in quanto elemento tecnico del discorso politico, tale rapporto, almeno nella storia dell'Occidente, è in relazione con le peculiari categorie di pensiero su cui la stessa politica si fonda ( $c f r$. A. Cavarero, Corpo in figure. Filosofia e politica della corporeità, Milano, 1995, p. 8).

${ }^{2}$ Non è questa la sede per una ricognizione di significati e teorie antiche sulla retorica. L'unico richiamo specifico da fare riguarda i collegamenti tra l'arte del bel parlare e del persuadere, come finalità alte del linguaggio che tende al coinvolgimento della mente, ma anche a suscitare l'emozione degli ascoltatori, e la partecipazione fisica. La stretta connessione tra res e verba, in quanto fondanti il potere dell'oratore, costituisce una innovazione di Cicerone, che dava a essa valore etico, in opposizione alla sofistica. Ma, ancor più innovativo, nonché antesignano di una tradizione culturale ininterrotta e con molteplici riprese è il rapporto tra actio e pronunciatio, dove «il gesto assume una funzione di supporto della voce secondo i diversi gradi dell'intonazione e il significato del discorso» (cfr. E. Agazzi, Il corpo conteso: rito e gestualità nella Germania del Settecento, Milano, 2000,
} 
sentita oggi da una scienza che, nel corpo, trova lo snodo flessibile per una ricerca fondata su trasversalità e interdisciplinarità dei saperi.

Tutte le forme di comunicazione conoscono un registro retorico, che può operare sia sul piano verbale che su quello visivo o più propriamente fisico, in relazione a comportamenti e a un linguaggio del corpo che si può estrinsecare anche in espressioni avulse dalla comunicazione verbale ${ }^{3}$.

Il corpo, del resto, è strumento di relazione fondamentale: oltre che rispetto all'identità individuale, esso è il luogo fisico in cui si rispecchia ogni relazione o rappresentazione umana, è strumento di definizione identitaria, è il confine tra noi e gli altri, è mezzo di comunicazione relazionale. In tutte le relazioni private e pubbliche, interpersonali, affettive e di potere, il corpo è anche strumento di comunicazione metalinguistica, di comunicazione non verbale $(\mathrm{CNV})^{4}$ : attraverso di esso il messaggio passa non solo come completamento e integrazione della parola, accompagnando con il gesto un pensiero intellettuale e consapevole, ma anche oltre la volontà cosciente, come gesto, istintivo, spontaneo, come atto automatico o riflesso, o, ancora, eterodiretto.

Oltre la suggestione ciceroniana e la tradizione retorica classica, che collocavano il corpo solo all'interno di un sistema educativo e formativo, e lo ritenevano importante in quanto funzionale per l'uomo politico nel suo ruolo pubblico ${ }^{5}$, oggi sappiamo che il corpo, anche nella dimensione relazionale, è molto di più: crocevia e strumento di un potere dalle molteplici forme e implicazioni, esso interagisce nelle dinamiche di potere presenti in tutte le relazioni, posto che non solo le relazioni politiche, ma anche quelle private, possono scivolare verso tali dinamiche ${ }^{6}$.

Nella riconsiderazione delle relazioni tra corpo e linguaggio, intendiamo qui analizzare non quelle forme di comunicazione che riguardano l'uomo di potere, in

p. 29). L'actio, già anticipata da Aristotele, come risvolto emozionale di ogni discorso, scritto o orale, con Cicerone diventa l'aspetto dinamico che alla fine riassorbe l'opera scritta, attraverso la declamazione e il coinvolgimento del corpo ( $c f r$. J. Di Bessière, E. Kushner, R. Mortier, J. Weisgerber (eds.), Storia delle poetiche occidentali, trad. it., Roma, 2001, p. 128; A. Tedeschi, Lezione di buon governo per un dittatore: Cicerone, 'Pro Marcello': saggio di commento, Bari, 2005, pp. 20ss.; E. Narducci, Cicerone: la parola e la politica, Bari, 2009, p. 286; B. Puca, Una periautologia paradossale: analisi retorico-letteraria di Gal 1,13-2-21, Roma, 2011, p. 25; e, da ultimo, B. Mortara Caravelli, Manuale di retorica, Milano, 2012).

${ }^{3}$ Studi contemporanei sulla pubblicità sembrano riferire solo a quest'ultima -figlia degenere della retorica persuasiva (Fabris)- l'applicazione di elementi di una retorica visiva ( $c f r$. A. Gruni, Le figure retoriche in pubblicità. Teoria e applicazione al quadrato semiotico di Floch, tesi di laurea, http://www.tesionline.it/ consult/brano.jsp?id=4767; R. Stivali, «Barack Obama e l'arte di persuadere: tra strategie retorico-linguistiche e linguaggio del corpo», http://www.englishfor.it/rivista_articolo4_1_09.asp).

${ }^{4}$ Una prospettiva in tal senso è il portato soprattutto della psicologia, e ha conosciuto un notevole sviluppo nella seconda metà del Novecento. Essa si occupa di tutti quegli elementi estranei al linguaggio, che ci permettono di comprendere qualcosa di una persona e possono comunicare significati personali e sociali di grande rilevanza. Fondamentale, a tale proposito, è M. Argyle, Il corpo e il suo linguaggio. Studio sulla comunicazione non verbale, Bologna, 1992.

${ }^{5}$ M. Foucault, La cura di sé. Storia della sessualità, III, trad. it. Milano, 1991, soprattutto il cap. Il gioco politico, pp. 83 ss.

${ }^{6}$ Senza addentrarci in questa questione, ricordiamo soltanto che la psicologia legge in chiave di potere anche le dinamiche interpersonali, interpretabili in tal senso anche attraverso la linguistica e la metalinguistica, in quanto linguaggio del corpo ( $c f r$. R. Segatori, L'ambiguità del potere: necessità, ossessione, libertà, Roma, 1999). 
quanto servono ad acquisire e gestire consenso con l'arte del discorso e il gesto che lo accompagna ${ }^{7}$. Sempre restando nell'ambito specifico della sfera politica, intendiamo piuttosto analizzare in quale modo il potere pieghi a strumento di comunicazione il corpo di coloro sui quali lo stesso potere si esercita. Il contesto è quello dei riti di penitenza nel cristianesimo antico: esperienze che stanno lungo una linea di confine tra funzione politica del corpo da un lato e manifestazioni emozionali dall'altro, tra tecnica e artificio da un lato e spontaneità dall'altro.

L'interesse di questi riti sta nel fatto che le manifestazioni di pentimento e di mortificazioni dell'anima, che ci aspetteremmo fossero solo espressione spontanea di interiorità spirituale, si trovano, invece, a doversi modellare su preordinati stilemi di comunicazione che rispondono alle richieste del potere ecclesiastico. In tal senso, il messaggio di pentimento che essi danno sta lungo una linea di comunicazione retorica.

Entro un percorso sulle forme di comunicazione, riconsiderando il corpo come linguaggio nella specifica dinamica di potere tra istituzione e individuo ${ }^{8}$, la nostra analisi incrocia gli studi sul tema del corpo $^{9}$ e della penitenza nel cristianesimo antico ${ }^{10}$.

Nel cristianesimo, il tema del corpo, strutturato in modo fondante sulla teologia del Verbo incarnato, e, dunque, da sempre collegato a riflessioni teologiche e antropologiche, ha trovato una rinnovata attenzione storiografica, a partire dagli anni '70, soprattutto per le problematiche riguardanti la storia della sessualità. Dopo secoli di silenzio storiografico ${ }^{11}$, la scoperta del corpo come possibile oggetto di indagine storica, soprattutto all'interno del tema del controllo sessuale come chiave di interpreta-

\footnotetext{
${ }^{7}$ Sempre in riferimento alle origini della questione e a Cicerone, $c f r$. C. Novelli, La retorica del consenso: commento alla tredicesima Filippica di M. Tullio Cicerone, Bari, 2001.

${ }^{8}$ Ricca di stimolanti ricerche in tal senso è la medievistica. Ricordiamo soltanto i fondamentali M. Bloch, I re taumaturghi. Studi sul carattere soprannaturale attribuito alla potenza dei re soprattutto in Francia e in Inghilterra, pref. C. Ginzburg, e un Ricordo di Marc Bloch di L. Febvre, trad. it., Torino, 1973. Classico sull'uso del corpo -sia pur dopo la morte-e sulle potenzialità comunicative che esso può comunque indurre in funzione del potere è A. Paravici Bagliani, Il corpo del papa, Torino, 1994. Del resto il corpo del capo, in quanto strumento di comunicazione e di potere, ha indotto riflessioni per qualunque epoca ( $c f r$. F. Boni, Il corpo mediale del leader: rituali del potere e sacralità del corpo nell'epoca della comunicazione globale, Roma, 2002). Nell'area di una riflessione sulla comunicazione attraverso il corpo riguardante tutti, non solo il capo, per l'antichità cristiana, si possono citare gli studi sul riso. In questo ambito, infatti, in quanto il riso è controllato e represso, si realizza una forma di controllo e di potere in una dinamica di tipo culturale e che rinvia a una visione antropologica su un registro speculare a quello dell'indagine qui affrontata (tra questi studi citiamo soltanto C. Mazzucco, (ed.), Riso e comicità nel cristianesimo antico. Atti del convegno di Torino 4-6 febbraio 2005, Alessandria, 2007).

${ }^{9}$ Numerosissimi gli studi in tal senso, per i quali ricordiamo solo i classici: M. Foucault, Storia della sessualità, 3 vols., Roma, ed. Feltrinelli, 2004, 2006, 2008; A. Rousselle, Sesso e società alle origini dell'età cristiana, trad. it., Bari, 1985; P. Brown, Il corpo e la società. Uomini, donne e astinenza sessuale nei primi secoli del cristianesimo, trad. it., Torino, 2010.

${ }^{10}$ Cfr., per esempio, C. Vogel, Le pécheur et la pénitence dans l'église ancienne, Paris, 1965; P. Saint-Roch, La pénitence dans les conciles et les lettres des papes des origines à la mort de Grégoire Le Grand, Città del Vaticano, 1991, e, tra gli ultimi, A. Carpin, La penitenza tra rigore e lassismo. Cipriano di Cartagine e la riconciliazione dei lapsi, Bologna, 2008; e il saggio critico, a introduzione di Tertulliano di A. Carpin (ed.), La penitenza, Bologna, 2011.

${ }^{11}$ Cfr. P. Sorcinelli, «Il corpo che la storia ha dimenticato», Storia e futuro. Rivista di storia e storiografia 23 (2010) (http://www.storiaefuturo.com/it/numero_23).
} 
zione delle relazioni sociali, ha incentivato riletture che riconsiderano soprattutto il ruolo della donna, la famiglia, l'ascetismo e il monachesimo, il celibato ecclesiastico.

Allo stesso modo anche gli studi sulla penitenza ecclesiastica di età antica non contemplano ricerche come quella qui portata avanti. Alla penitenza sono stati dedicati studi sulla trattatistica e, dunque, dominati da questioni etiche e teologiche. $\mathrm{E}$, là dove si è preso in considerazione l'aspetto più propriamente giuridico e sanzionatorio, se ne sono cercati rapporti e quantificazioni con la gravità della colpa.

Fondamentale per la salvezza e la redenzione, la penitenza impone e consente al peccatore di esprimere, con linguaggio del corpo, la condizione della sua anima, lo stato di pentimento. Tempi, forme e modi di questa comunicazione -che non può avvenire che attraverso la fisicità del corpo e delle sue forme espressive- sono, però, dettati dalle norme ecclesiastiche. Avviene così che il peccatore, per convincere dell'autenticità del suo pentimento, debba esprimersi secondo un linguaggio del corpo piegato dalla retorica del protocollo penitenziale, e che la spontaneità di un percorso religioso ed interiore, come dovrebbe essere il pentimento, sia piegata all'artificio di un rituale liturgico.

Le persecuzioni e ancor più la loro fine aprirono un periodo di resa dei conti tra chi aveva resistito e chi, abiurando in vario modo, aveva ceduto al vincolo di fedeltà e di testimonianza nella fede in Cristo. Ne derivò una forte accelerazione nella riflessione sulla colpa e sulla resposabilità derivante da un'azione delittuosa compiuta sotto la pressione di una violenza subìta. Il corpo, attraverso cui si realizzano sia l'espressione di fede coerente ed estrema, fino all'effusio sanguinis, sia l'azione delittuosa dell'abiura, diventa anche strumento di comunicazione per capire la reale portata della colpa compiuta; esso consente anche, attraverso la marginalizzazione fisica -la scomunica o altre temporanee o ridotte forme di esautoramento dalla comunità- la pubblicizzazione della punizione; infine, attraverso adeguate e imposte forme di penitenza, è lo strumento di comunicazione di un pentimento attraverso il quale ottenere la reintegrazione anche fisica nella comunità.

\section{IL CORPO E LA COLPA: FORME ED ESPRESSIONI}

Nella cultura contemporanea, l'esperienza della colpa, assorbita in gran parte dalla psicanalisi che la ha condensata, però, nel suo senso più propriamente psicologico, non si esaurisce in essa. La colpa si muove su diversi registri che riflettono la sua natura, che è, insieme, soggettiva e oggettiva, agita tra pensiero e azione, tra anima e corpo, tra etica e diritto. In ogni caso, l'esperienza della colpa si muove all'interno di meccanismi relazionali, dove il linguaggio, la comunicazione sono imprescindibili.

Nell'antica età cristiana, il corpo e le sue potenzialità di comunicazione sono fonte e genesi della colpa il primo, strumenti indispensabili per verificare natura e portata della colpa le seconde. Questa chiave di lettura si colloca lungo un percorso interpretativo partito da lontano, quando, nell' antichità greco-romana, il concetto di colpa rispecchiava una grande ambiguità. Pur con qualche sfumatura di interpretazione, 
in età arcaica e classica, tutti gli eventi erano in mano a dio, a démoni o a spiriti ${ }^{12}$. Un'azione sbagliata era da rinviare alla loro collera, il colpevole era solo un demens, preda di un delirio o di una pazzia; il suo delitto era un errore involontario, di cui non era direttamente responsabile ${ }^{13}$. Eschilo e Sofocle iniziarono a porsi il problema della responsabilità del soggetto rispetto all'azione, ma non si emanciparono dall'ambiguità della colpa che colorava la tragedia. L'oggettività della trasgressione era evidente, ma era incerta la possibilità di una imputazione precisa e certa. Il concetto di responsabilità restava del tutto estraneo nella classicità greca ${ }^{14}$.

Il concetto di responsabilità appartiene a una famiglia semantica che comprende campi di azione che vanno dalla morale al diritto alla religione. In sé, idea e nozione di responsabilità appartengono all'età moderna e derivano dall'etica kantiana ${ }^{15}$. Peraltro, nel mondo antico, tale nozione emerge da un contesto complessivo ${ }^{16} \mathrm{e}$, là dove essa si fa strada, come in Platone e Aristotele, non sempre è chiaro il discrimine tra responsabilità morale e responsabilità giuridica, o essa è poco approfondita ${ }^{17} . \mathrm{Nel}$ mondo romano, per esempio, il cittadino risponde a una teoria organicistica dello stato, di cui è parte integrante e per la quale si sente responsabile; pure restando nell'ambito del diritto, in riferimento alla responsabilità, tale teoria richiama i principi etici fondamentali del neminem laedere. Ma, è con ebraismo e cristianesimo che sembra configurarsi lo stato nascente della soggettività, con i suoi elementi costitutivi di compiuta riflessività, autonomia della scelta, consapevole originalità della persona ${ }^{18}$.

In questo percorso, che individua nel cristianesimo passaggi significativi nella definizione della responsabilità individuale, la prima documentazione conciliare rappresenta un momento rilevante. In essa, infatti, il principio della responsabilità etica, morale e religiosa di fronte a Dio si salda con quello della responsabilità giuridica: il

${ }^{12}$ La ü $\beta \rho 1 \varsigma$ dei greci ha natura ambigua e si colloca tra il male oggettivo ed esterno, il miasma, e la demenza ( $c f r$. le fondamentali pagine di E.R. Dodds, Civiltà di vergogna e civiltà di colpa, in I Greci e l'irrazionale, trad. it., Firenze, 1973, pp. 33-74.)

${ }^{13}$ R. Mondolfo, La comprensione del soggetto umano nell'antichità classica, Firenze, 1958, pp. 401-402, si riferisce alla tesi di Gernet quando afferma che nella Grecia antica la nozione di «crimine oggettivo» precede quella di «soggetto criminale».

${ }^{14}$ In contrasto con C. Del Grande (Colpa e castigo nell'espressione poetica e letteraria degli scrittori dell'antica Grecia tra Omero e Cleante, Napoli, 1947) e Mondolfo, così S. Natoli, L'esperienza del dolore. Le forme del patire nella cultura occidentale, Milano, 2002, p. 56, n. 6, il quale richiama il mito di Prometeo quale testimonianza dell'inevitabilità del delitto. Così anche A. Jellamo, Il cammino di Dike: l'idea di giustizia da Omero a Eschilo, Roma, 2005, p. 41: «[...] i personaggi omerici possono essere colpevoli, ma non responsabili $[\ldots] \gg$.

15 G.P. Calabrò (ed.), La nozione di responsabilità tra teoria e prassi, Milano, 2010, pp. 17-18. Resta fuori dai nostri riferimenti un altro ambito fondamentale in cui la cultura contemporanea sviluppa il concetto di colpa, quello del registro in chiave di soggettività e interiorità individuale -il senso di colpa, di cui parla la psicanalisi-.

${ }^{16}$ M. Zanatta, Figure della responsabilità nel mondo greco, M. Zanatta-B. Franco (ed.), Responsabilità e comunità, Cosenza, 2007, p. 10, indica significative riflessioni nel senso della responsabilità in Platone e Aristotele.

${ }^{17}$ Cfr. J.P. Vernant-P. Vidal Naquet, Mito e tragedia nell'antica Grecia, trad. it., Torino, 1976, a proposito di Aristotele.

${ }^{18}$ V. Cesareo-I. Vaccarini, La libertà responsabile. Soggettività e mutamento sociale, Milano, 2006, pp. 83 ss.; V. Lusetti, Il cannibalismo e la nascita della coscienza, Roma, 2008, pp. 182 ss. 
che è anche il portato più specifico del diritto canonico ${ }^{19}$, formulazione teologico-giuridica attraverso la quale la comunità cristiana, come ogni società umana e/o religiosa, si regolamenta con un sistema di leggi che, nella implicita possibilità che le indicazioni vengano disattese ${ }^{20}$, sono accompagnate da diversi modi e gradualità di sanzioni. Peraltro, si tratta di una situazione non generalizzata: non tutti i sistemi giuridici diversificano le sanzioni, stabilite in base a una più o meno attenta considerazione della gravità della colpa, né tutti, come abbiamo detto, pongono il problema della responsabilità umana rispetto alla colpa o al peccato né tutti diversificano tra colpe di tipo ideologico o reati corrispondenti ad azioni concrete.

Fu nel cristianesimo che si assisté a una forte incentivazione della riflessione sul tema della responsabilità di fronte al peccato, quando, in seguito ai decreti imperiali, i cristiani vennero costretti ad atti di abiura e, alla fine delle persecuzioni, i lapsi vennero sottoposti al giudizio delle comunità e dei vescovi, riuniti in sinodi.

Si pose in dubbio la possibilità di esprimere un giudizio di condanna certo e giusto nei confronti di un cristiano che avesse sacrificato perché sottoposto a violenza. Si ritenne necessario valutare in quali condizioni questo sacrificio fosse avvenuto, se vi era stata costrizione o meno da parte delle autorità romane. L'approfondimento, nella documentazione conciliare teoreticamente non molto sviluppato ma consapevole nella natura delle deliberazioni, si sviluppò tra conflitti interni di natura disciplinare e scontri sulla necessità di trattare più o meno severamente i lapsi. La questione del rapporto tra peccato, condanna e possibilità di salvezza ha conosciuto momenti durissimi di confronto-scontro, con una forte concentrazione dialettica in alcune fasi. Gli esiti storici più eclatanti sono connessi alle derive scismatiche, con le note conseguenze legate alla vicenda di Novaziano e a quella, divenuta contingua a più complesse questioni dottrinali, dell'arianesimo.

A livello conciliare, il problema di capire l'effettivo coinvolgimento della volontà nel peccato divenne concreto e pressante perché si potessero stabilire forme e modi della penitenza ${ }^{21}$.

I vescovi riuniti al concilio di Ancira (314), chiamati a decidere interventi disciplinari in relazione a quei fedeli, lapsi, che avessero variamente abiurato ${ }^{22}$, stabilirono che non era sufficiente che un cristiano avesse sacrificato per esprimere un giudizio di condanna. Era necessario valutare in quali condizioni questo sacrificio fosse avvenuto, se vi era stata costrizione fisica o meno. Una colpa commessa in conseguenza di una violenza subita -come è appunto nel caso del cristiano costretto a una

\footnotetext{
${ }^{19}$ Sulla specificità dei rapporti tra diritto divino e diritto umano, che contraddistinguono il diritto canonico, fondamentali le analisi teoriche nelle varie opere di J. Gaudemet, di cui una sintesi è Il diritto canonico, trad. it., Milano, 1991.

${ }^{20}$ Sulla differenza tra sistemi giuridici che configurano significati e forme diverse di disobbedienza, quali il reato e il peccato, $c f r$. le pagine introduttive in P. Prodi, Una storia della giustizia: dal pluralismo dei fori al moderno dualismo tra coscienza e diritto, Bologna, 2000.

${ }^{21}$ Sulle caratteristiche di povertà documentaria, nei testi penitenziali antichi, $c f r$. Saint- Roch, La pénitence, cit., pp. $17 \mathrm{~s}$.

${ }^{22}$ La posizione degli ecclesiastici è distinta da quella dei laici e non è qui considerata nella sua specificità.
} 
dichiarazione di abiura nelle sue varie forme, dal rinnegare verbalmente Dio al sacrificare o bruciare incenso- andava meglio approfondita, andava sondato il limite tra coscienza e volontà da un lato e azione compiuta dall'altro. A dirimere queste discriminanti solo i messaggi del corpo: a questi si attribuiva implicitamente il valore di un codice semantico e, per questo, dovevano essere decodificati come un linguaggio.

Il giudizio sulla condotta passava attraverso quanto comunicava il corpo durante, ma anche dopo la violenza subita. Durante e dopo la violenza il fedele doveva comunicare in modo adeguato di essere estraneo a quanto aveva compiuto:

Coloro che mentre erano in fuga furono catturati oppure furono traditi dai servi o subirono la confisca dei loro beni oppure furono sottoposti ai tormenti o furono messi in carcere, poiché proclamavano di essere cristiani e, una volta incarcerati, sono stati costretti a prendere nelle loro mani (l'incenso per gli idoli) o hanno mangiato per costrizione un qualche cibo (offerto agli idoli), continuando, comunque, a confessare di essere cristiani, e che mostrano sempre il dolore di quanto è loro accaduto con ogni modestia, comportamento e umiltà di vita, a costoro non si proibisca la comunione, dal momento che non hanno commesso peccato; se è stato proibito loro da qualcuno per eccessiva cautela o per ignoranza siano subito reintegrati. Lo stesso vale similmente per i membri del clero e per gli altri laici. E' stato preso in considerazione anche questo: se i laici caduti per la stessa costrizione possano accedere all'ordine sacro, è stato deciso che costoro, dal momento che non hanno peccato, siano promossi, a condizione che anche il comportamento precedente della loro vita venga trovato corretto ${ }^{23}$.

Rispetto alle pratiche di culto imposte e alla partecipazione ai banchetti sacrificali, dovevano essere tenuti in considerazione il linguaggio e la comunicazione del corpo: era necessario considerare l'aspetto, il portamento, il comportamento e il modo di vestire dei lapsi e solo nel caso non avessero dimostrato ripulsa andavano adeguatamente allontanati:

Riguardo a coloro che hanno immolato agli idoli per forza ed hanno preso parte anche ai banchetti in occasione dei sacrifici; se costoro, quando furono presi, entrarono nei templi con aspetto piuttosto felice e si misero abiti piuttosto eleganti e parteciparono al banchetto preparato senza farsi problemi, è stato deciso che per un anno siano posti tra gli uditori, per tre anni saranno tra i penitenti, per due anni possano comunicare solo nella preghiera e solo allora giungano alla perfezione della grazia $^{24}$.

Andava, inoltre, valutato se avevano spontaneamente partecipato ai culti, se «avevano partecipato ai banchetti durante una festa pagana o in un luogo destinato ai pagani

\footnotetext{
${ }^{23}$ Can. 3 (A. Di Berardino (ed.), I canoni dei concili della Chiesa antica, I. I concili greci (eds. C. Noce C. Dell'Osso-D. Ceccarelli Morolli), Roma, 2006, trad. it., p. 271).

${ }^{24}$ Can. 4, trad. it., p. 273.
} 
avendo portato e mangiato le proprie vivande» ${ }^{25}$; oppure se «erano stati costretti ad immolare agli déi due o tre volte» ${ }^{26}$, e, ancora, se «...salirono ai templi con vesti di lutto e mettendosi a tavola mangiarono piangendo per tutto il tempo in cui prendevano cibo...»o «se invece non mangiarono affatto» ${ }^{27}$. Qui, tutto è centrato sul corpo. La violenza è ritenuta dimostrata dal linguaggio del corpo. Questo comunica la partecipazione oppure la violenza subita, in ragione della quale la colpa dell'abiura ha un peso minore e, dunque, può essere ridimensionata anche rispetto alla punizione.

All'interno di una problematica più generale e che è oggetto di interesse sin dalle origini -la connessione tra teologia, antropologia e soteriologia- si impone una valutazione della natura e della gradualità dei peccati ${ }^{28}$. Soprattutto, si apre una riflessione sulla volontà di chi li commette e sui rapporti tra volontà e colpevolezza, premessa inevitabile per ogni discorso sul pentimento, sulla penitenza e sulla salvezza ${ }^{29}$.

Nel confrontarsi con il problema del rapporto tra corpo, volontà e peccato facendo i conti con le violenze subite, si pose la questione di stabilire il grado di responsabilità nel rapporto tra azione e volontà ${ }^{30}$. In questa questione, il corpo diventa un confine da superare rispetto a quello che fa e che rappresenta, le azioni che gli fanno riferimento sono segni di un linguaggio da interpretare, per riallacciare la connessione tra corpo e anima, e ricostruire il vero legame tra pensiero e azione. Definire il giusto rapporto tra volontà e peccato diventa una necessità in vista della punizione. Il tema scritturistico del peccato che deriva dalla corruzione del cuore ${ }^{31}$ spinge a precisare la portata di quest'ultima prima di indicare la condanna.

In questo contesto, il corpo diventava confine e linguaggio, strumento con cui il delitto era stato commesso; ma, in quanto frontiera dell'anima, forniva anche messaggi di innocenza o di responsabilità solo parziale. La costrizione -costrizione psi-

${ }^{25}$ Can. 7., trad. cit., p. 273.

${ }^{26}$ Can. 8, trad. cit., p. 275.

27 Can. 5, trad. cit., p. 273.

${ }^{28} \mathrm{Su}$ questi, cfr. Vogel, op. cit., soprattutto pp. 13 ss.

${ }^{29}$ Nei trattati sulla penitenza, anche vicini alle problematiche delle persecuzioni, come quelli di Tertulliano (De poenitentia 3 ) e Cipriano, l'attenzione è sul valore morale, sulla opportunità e necessità della penitenza in vista della riconciliazione. In Ambrogio sono fondamentali le questioni teologiche e antropologiche sulla redenzione e salvezza, i temi del peccato e della colpa umana, la possibilità/necessità del perdono e, quindi, quella di stabilire la penitenza, la funzione della preghiera, il ruolo e il potere in tal senso dei ministri della Chiesa, il dibattito, su tutte queste questioni, con altre chiese e dottrine. Largo spazio ha la riflessione sulla violenza e sulla necessità del perdono per i lapsi violentati e costretti: 1, 6, 25-26: qui anche il diavolo sentenzia: «Cristo non subisce danno quando persone venute da me in lacrime ritornano nel seno della Chiesa, sentendo di essa rimpianto» (Ambrogio, La penitenza. trad., int., e n., E. Marotta, Roma, 1976, p. 47).

${ }^{30} \mathrm{Il}$ tema dell'etica e della responsabilità, in termini morali, è strutturato o sotteso in vario modo a Scritture e testi dei Padri. Qui, si evoca piuttosto la questione della separatezza tra le azioni compiute e la condizione interiore di ascendenza evangelica ( $c f r$. Mt 15, 11-21; Mc 7, 1-23). Inoltre, il testo presuppone un ulteriore approfondimento teorico perché chiama in causa la dialettica tra azione, volontà e violenza centrando sul tema della responsabilità rispetto a un'azione compiuta per violenza subìta e dunque scorpora il concetto di errore/peccato rispetto alla colpa. Filosofia del diritto e giurisprudenza hanno variamente indagato la questione. $\mathrm{Ma}$, nel cristianesimo, il problema è più complesso anche per gli esiti che il commettere un'azione peccaminosa ha sull'anima del peccatore. Cfr. Agostino: «La passione ha origine dalla volontà perversa» $($ Conf. 8, 5).

${ }^{31}$ Il riferimento è a Gen 6,$5 ;$ Is 29, 13; Ger 17, ma non è esplicito. 
cologica o fisica, fatta o subìta- entra in più modi, anche come elemento di semplice pressione sui compagni di fede, a creare discrimini di colpa.

Per esempio, si considerano i casi di coloro che «non solo hanno apostatato, ma anche si sono levati contro i fratelli ed hanno costretto anche loro ad apostatare, essendo divenuti la causa della costrizione di altri ${ }^{32}$.

$\mathrm{O}$, ancora, la violenza fisica subita in concreto distingue la condizione di coloro che, per l'appunto, ne sono stati vittime da coloro che, invece, hanno ceduto alla sola minaccia di violenza, dimostrando maggiore debolezza e fragilità di fede ${ }^{33}$.

Nei concili, la colpa è dimostrata dal comportamento e dall'atteggiamento del corpo: è questo che commette l'azione peccaminosa, ed è questo che dimostra l'effettiva adesione o non adesione all'atto compiuto. E' questo ad essere l'unico mezzo per conoscere l'anima. E se ha subìto violenza, la fragilità del corpo impone comprensione nei confronti delle vittime: per calibrare l'indulgenza e ridimensionare la sanzione, è necessario ascoltare/guardare linguaggio e segni del corpo.

Un cristiano che compisse un sacrificio commetteva un sacrilegio ed era da punire severamente. Ma, costrizione e violenza subita e comprovata comportavano colpa meno grave $\mathrm{o}$, a volte, anche nessuna colpa ${ }^{34}$. Una volontà forzata a compiere con il corpo un'azione delittuosa, dunque, può non contaminare l'anima. In taluni casi, infatti, non c'era nemmeno colpa e, dunque, poteva non esserci nemmeno necessità di perdono. Attraverso il corpo, la violenza subita dalla volontà e dalla coscienza comunicava l'innocenza dell'anima, lasciava intatta la condizione di incolpevolezza ${ }^{35}$. L'anima, data la sua reale disposizione, non veniva segnata dalla colpa e dalla condanna, perché il corpo, cedendo alla debolezza, si poneva in ultima analisi quale frontiera e confine della coscienza.

Affermare che la violenza subìta non comporta nessuna colpa non è assolutamente una ovvietà, come dimostrano dibattiti e atteggiamenti contemporanei al nostro testo, ma anche nella moderna e contemporanea cultura giuridica ${ }^{36}$.

Il tema della libera volontà come unico elemento di discrimine tra il bene e il male non è sufficiente a coprire tutti gli aspetti in cui anche in questo contesto il corpo continua a essere chiamato in causa per testimoniare che la volontà rinnegava l'azione sacrilega nello stesso momento in cui essa veniva compiuta.

La violenza, comprovata nel momento in cui è subìta, da sola, non è sufficiente a dimostrare la non colpevolezza. E una volontà forzata a compiere un'azione delittuosa non contamina l'anima purché il corpo continui a esprimere con il proprio linguaggio e attraverso le proprie specifiche forme di comunicazione l'estraneità di

\footnotetext{
32 Can. 9, trad. cit., p. 275.

${ }^{33}$ Can. 6.

${ }^{34}$ Can. 3: .«..dal momento che non hanno peccato» (trad. cit., p. 271).

35 A. Rousselle, op. cit.; F. Lucrezi, La violenza sessuale in diritto ebraico e romano, Milano, 2004, p. 31.

${ }^{36}$ La storia dello stupro violento nella cultura giuridica europea dal XVIII secolo a oggi lo dimostra: la violenza subìta non salvaguarda la vittima, anche al di là di ogni discrimine e valutazione sulla verifica che effettivamente di violenza si sia trattato. Infatti, giuristi, politici e medici legali hanno considerato la violenza sessuale un reato contro la morale e, il più delle volte, una colpa della vittima ( $f f r$. T. Noce, Il corpo e il reato. Diritto e violenza sessuale nell'Italia dell'Ottocento, S. Cesario di Lecce, 2009).
} 
tutto l'essere rispetto a quanto compiuto. Così, condizione di colpevolezza o innocenza, in ragione della dissociazione della volontà dall'azione sacrilega compiuta, dovevano essere testimoniati in successive manifestazioni anche dopo il supplizio, e, ancora una volta, unico mezzo per comunicarli, erano i segni e il linguaggio del corpo $^{37}$.

I segni di violenza fisica e psicologica comunicati dal corpo che, nella letteratura conciliare, consentivano di calibrare la colpa in funzione del giudizio, hanno, negli Atti dei martiri, un ruolo complementare e speculare. Le condizioni di violenza che, nella letteratura conciliare, sono condizioni che servono a ridimensionare il peccato, negli Atti, ridimensionano, specularmente, anche il senso dell'esperienza martiriale e riducono il valore del martirio nel suo senso più proprio di testimonianza.

Con la consapevolezza che un atto di abiura forzato possa non conformarsi a un adeguato atteggiamento della coscienza - coscienza che, nella logica dei persecutori, deve essere realmente piegata perché l'abiura abbia un senso reale-, nel martirio di Policarpo, il proconsole, prima delle minacce di terribili tormenti, vuole imporre al vescovo una esplicita e verbalizzata dichiarazione di abiura: «Giura sulla fortuna dell'imperatore» «Pentiti» ${ }^{38}$.

La richiesta del proconsole riflette la considerazione che ciò che conta è piegare la coscienza ${ }^{39}$ e che la parola -come linguaggio verbale- esprima adeguatamente la propensione della coscienza, ne registri fedelmente un reale ripiegamento: per questo, talvolta, la dichiarazione di abiura sembra avere più valore di un atto di culto imposto. Così, le torture fisiche servono per ottenere l'abiura verbale.

Nell'interazione e comunicazione fisica che si stabilisce tra la violenza attiva perpetrata dai persecutori e la forza resistente del cristiano, che reagisce alla violenza subìta, il corpo è l'ultima barriera da abbattere, il confine superato il quale i persecutori hanno maggiori chance di successo per piegare la coscienza dei perseguitati. L'abiura intellettuale è il fine ultimo, ma per ottenere questa abiura intellettuale, i procuratori romani fanno leva sulla pressione psicologica e sul terrore delle sofferenze fisiche, reali o minacciate ${ }^{40}$. Pur di ottenere l'abiura, i persecutori operano con mellifui tentativi di convinzione o minacce ${ }^{41}$.

\footnotetext{
${ }^{37}$ Can. 3: «[...] continuando, comunque, a confessare di essere cristiani, e che mostrano sempre il dolore di quanto è loro accaduto con ogni modestia, comportamento e umiltà di vita [...]» (trad. cit., p. 271).

${ }^{38}$ Martyrium Carpi 9, 2, (Atti e Passioni dei martiri. Intr., A.A.R. Bastiaensen, ed., A.A.R. BastiaensenA.Hilhorst-G.A.A. Kortekaas-A.P.Orbán-M.M. van Assendelft (trad. S. Ronkey), Milano, 1987, trad. it., p. 17. Da notare anche l'utilizzo capovolto del verbo pentirsi.

${ }^{39}$ Il coinvolgimento della coscienza negli atti di culto e, dunque, in contesto religioso, non appartiene alla dimensione della religiosità antica, così come mancava quello di libertà religiosa: ma, età ellenistica e mondo tardoantico si aprirono a una sensibilità più dinamica dove comparve la coscienza religiosa intesa come rapporto intimo e personale con la divinità: per un confronto ravvicinato tra esperienze di religiosità pagane e cristiane che coinvolgono il tema della coscienza, cfr. T. Sardella, Pregare gli dei/pregare Dio. La preghiera come fattore identitario tra pagani e cristiani: i casi di Elio Aristide e Policarpo, G. Ruggieri (ed.), La Preghiera. Manifestazione elo fattore d'identità, Catania, 2012, pp. 11-38.

${ }^{40}$ Martyrium Carpi 9.

${ }^{41}$ Martyrium Policarpi 8, trad. cit., p. 15: «Che c'è di male a dire Cesare Signore, e a compiere il sacrificio $[\ldots]$ ?.»
} 
In taluni casi, minacce e violenze tendono come scopo unico a rintuzzare la professione di fede cristiana, reiteratamente proclamata dal cristiano e a ottenere che egli compia il sacrificio agli dei o all'imperatore come atto di abiura ${ }^{42}$.

Il corpo o i gesti del corpo, dunque, sono strumento utile per piegare il fedele, in essi sono riflesse la forza e la volontà dell'anima: è questa che lotta nel corpo suppliziato ed è questa che i persecutori vogliono piegare: «Il beato Potino ... rinvigorito dall'empito dell'animo suo che ardentemente bramava il martirio, lasciò che lo traducessero davanti al tribunale, il corpo affranto dalla vecchiaia così come dalla malattia, eppure l'anima entr'esso battagliava, perché attraverso di lei Cristo trionfasse ${ }^{43}$.

In conclusione, la violenza subìta dà un significato diverso all'azione compiuta e riconduce a un suo ridimensionamento. Il sacrificio e ogni atto di abiura, se compiuti sotto pressione psicologica o violenza fisica, non comunicano una vera abiura -dell'anima-, hanno un valore diverso.

In contesto normativo, la necessaria verifica della colpa, per stabilire la sanzione per chi abbia abiurato sotto costrizione, impone di valutare meno gravemente la colpa e di ridimensionare la penitenza. Per converso, negli Atti dei martiri la violenza subita e non scelta sminuisce il senso del sacrificio di sé in quanto testimonianza, appare come un disvalore della stessa esperienza martiriale; riduce il valore della attestazione di fede, che è sacrificio reale solo se il martirio è, se non ricercato, almeno scelto e non imposto con la violenza e subito per paura.

In un clima di pura esaltazione del martirio, il sacrificio dei martiri cartaginesi -che non è l'atto di culto sacrilego compiuto dai lapsi, ma è il sacrificio di sè, l'atto attraverso il quale il martire si immola-, la effusio sanguinis, rende testimonianza estrema di fede, ma solo se affrontata non per paura, ma liberamente.

Così come per la colpa, anche per la ricompensa, il valore della violenza subìta in funzione della salvezza eterna sta nella pienezza della libertà: «Siamo giunti al martirio spontaneamente, proprio perché la nostra libertà non venisse incatenata ${ }^{44}$. La libertà è discrimine di grandezza sia rispetto alla colpa che rispetto al martirio. Il corpo ne traccia il confine.

\section{IL LINGUAGGIO DELLA SANZIONE: LA SCOMUNICA E LA 'ACTIO' RETORICA DELLA PENITENZA PUBBLICA}

La retorica, nella tradizione classica, ruotava attorno al linguaggio verbale. La quinta e ultima parte dell'elaborazione del discorso, detta actio, concernendo tutti gli aspetti

\footnotetext{
${ }^{42}$ Acta Iustini 5, 6; Martyrium Lugdunensium 1, 20.

${ }^{43}$ Martyrium Lugdunensium 1, 29, trad. cit, p. 75.

${ }^{44}$ Passio Perpetuae et Felicitatis, (Atti e passioni dei martiri, ed. cit., 18, 5, p. 140): Ideo ad hoc sponte pervenimus, ne libertas nostra obduceretur [...] (trad. cit., p. 141). Sulla ricerca del martirio volontario come valore, le testimonianze di età martiriale propongono sia il caso di Policarpo che quello di Cipriano, ma non sempre la Chiesa ha orientato in tale direzione il comportamento dei cristiani: cfr. A. Di Berardino, Il modello di martire volontario, in Euplo e Lucia. 304-2004, Agiografia e tradizioni cultuali in Sicilia. Atti del Convegno di Studi (Catania-Siracusa 1-2 ottobre 2004), T. Sardella-G. Zito (eds.), Catania, 2006, pp. 63-105.
} 
che il discorso pubblico può avere in comune con la recitazione, riguardava il linguaggio del corpo. La retorica classica, dunque, conosceva il problema del linguaggio del corpo, ma ne faceva pur sempre una componente della comunicazione verbale della quale il linguaggio del corpo era parte integrante.

In realtà, c'è anche una retorica del linguaggio del corpo, che è avulsa dalla comunicazione verbale alla quale la legava la retorica classica. Ma, è anche avulsa dalla CNV, ma spontanea, di cui parla la moderna semeiotica del corpo. Così alla necessità -conosciuta e riconosciuta- di decodificare il linguaggio del corpo, là dove esso si esprima in azioni spontanee, si aggiunge un linguaggio del corpo, codificato nelle forme, e la cui semantica appartiene ai codici del sistema di comunicazione del potere.

$\mathrm{Nel}$ cristianesimo, è ciò che avviene per sanzioni quali scomunica e penitenza. Gesti e parole di pentimento, imposti nei periodi di penitenza e scomunica, codificano dall'alto il linguaggio del corpo. Al peccatore si chiede di comunicare il proprio pentimento, al fine di ottenere reintegrazione e salvezza ${ }^{45}$, in periodi e forme diverse che coniugano diversamente uno stesso codice di uso e comunicazione del corpo da parte del potere, quello dell'emarginazione fisica del colpevole, respinto fuori dalla comunità.

Michel Foucault, in Sorvegliare e punire. Nascita della prigione ${ }^{46}$, si interroga «Da dove viene questa strana pratica, e la singolare pretesa di rinchiudere per correggere, avanzata dai codici moderni?». Messo a punto tra XVI e XIX secolo, il sistema della carcerazione punitiva è una innovazione della modernità.

Il sistema penale antico, infatti, conosce un registro della comunicazione del potere fondato sulla sanzione nel segno dell'espulsione - con l'eliminazione fisica del colpevole attuata in vari modi- piuttosto che della reclusione, dell'eliminare piuttosto che del mantenere.

La carcerazione avrà sempre per il sistema romano più che carattere di pena quello di misura preventiva. Ulpiano sottolinea infatti che «il carcere è destinato a custodire gli uomini, non a punirli». E la pena di morte resta nell'antichità il principale sistema di repressione messo in atto a partire da un fenomeno normativo, la cui origine è strettamente legata al pensiero religioso, dove anche forme e modi della repressione, soprattutto la pena di morte, mantengono un significato sacrale ben oltre l'età precivica ${ }^{47}$. La pena di morte, in tarda età repubblicana, sarà applicata raramente, e sostituita da pene pecuniarie o dall'esilio proprio per il decadere di una rigida concezione religiosa della pena. Caduta in disuso nel I secolo a. C., essa trova nuovo slancio in età imperiale ${ }^{48}$. Il

45 Sui rischi di perdita di salvezza, che comporta non seguire le sanzioni ecclesiastiche, $c f r$. T. Sardella, «Dalla scomunica all'Inferno. L'Al di là come strumento di potere nelle prime decretali», Sulle rive dell'Acheronte. Costruzione e percezione nella sfera del post-mortem (Velletri 12-16 giugno 2012). Atti del Convegno, sotto stampa.

${ }^{46}$ M. Foucault, Sorvegliare e punire. Nascita della prigione, trad.it., Torino, 1993.

${ }^{47}$ Le interdizioni rituali sono «il fondo della vita morale e giuridica delle società più semplici» (M. Mauss, I fondamenti di un 'antropologia storica, trad.it., Torino, 1998, p. 183): chi non osserva le interdizioni (tabu) deve essere punito con sistemi simili alla pena pubblica attuale. In questi contesti, manca un organo particolare che amministri la giustizia, la repressione è diffusa (F. Alonzi-R. Bonsignori, s.v. Pena, Universo del corpo, 2000, in www. Treccani.it).

${ }^{48}$ Accanto ai summa supplicia (per es., la crocifissione, l'esposizione alle belve e la vivicombustione), intesi quali pene a sé stanti e non come forme alternative di esecuzione della pena di morte (distinzione questa che 
mutamento del sistema processuale di Augusto porta a un diverso sistema di pene, articolate in modo graduato, di contenuto molto più afflittivo di quello tardo repubblicano. Come 1'esilio, insieme al principio della vendetta, la pena di morte mantiene il significato metagiuridico e sacrale di separazione sociale del colpevole dalla comunità, in funzione di purificazione e compensazione ${ }^{49}$.

In questo contesto di cultura giuridico-penale il cristianesimo dei primi secoli si trova a elaborare un proprio sistema punitivo. Inevitabile che le pratiche in uso potessero suggerire forme e metodi e che si ponga un problema di continuità/discontinuità rispetto alla percezione della punizione nella società romana di età imperiale.

Nei documenti normativi ecclesiastici del IV secolo compare come punizione diffusa e generalizzata la scomunica, in alternativa al meno diffuso anatema ${ }^{50}$. Pur con tutte le differenze del caso, ci pare che la scomunica, come formula punitiva, e valutando il messaggio primario insito nel suo meccanismo essenziale riflesso nell'etimologia latina ${ }^{51}$, possa essere ricondotta all'esilio, così come alla pena di morte. In altri termini, il codice di comunicazione punitiva messo in atto dal potere ecclesiastico è lo stesso insito nella natura repressiva del sistema penale antico che, riguardo alla punizione, tende ad allontanare piuttosto che a chiudere, ad espellere piuttosto che a

cadrà solo in epoca postclassica), le fonti menzionano altre pene che, sia pure non direttamente produttive di morte, sono equiparate (dai vari tipi di damnatio ai lavori forzati, a quelli accessori ecc., alla deportatio) e unite nell'effetto di porre il condannato nella condizione di servus poenae, privo cioè di ogni capacità giuridica. Questa tendenza, nettamente repressiva, alla creazione di nuovi crimina publica e alla loro punizione con la morte è il motivo dominante di tutta la legislazione imperiale ed espressione della volontà di uno Stato assoluto di avocare soltanto a sé il potere di punire ( $c f r$. Alonzi-Bonsignori, s.v. Pena, op.cit.).

${ }^{49}$ Nella discussione, tra storici del diritto, se l'esilio debba essere considerato l'esercizio di un diritto o una situazione penale, cfr. G. Agamben. «Politica dell'esilio», Derive Approdi. n. 16. Labirinto. Naples, 1998, p. $25:$ : $[.$.$] se l'esilio sembra eccedere tanto il repertorio dei diritti che quello delle pene, e oscillare tra l'uno$ e l'altro, ciò non è per una sua costitutiva ambiguità, ma perché esso si situa in una sfera per così dire più originaria, che precede questa partizione e in cui esso convive col potere giuridico-politico supremo. Questa sfera è, cioè, quella della sovranità, del potere sovrano») (cfr. G. Crifò, L'esclusione dalla città. Altri studi sull' exilium romano, Perugia, 1985, p. 136, n. 60).

${ }^{50}$ Oggi usati come sinonimi, i due termini, etimologicamente diversi, hanno significato nel tempo forme di condanna diverse ma anche simili tra loro. Inizialmente presso i Greci, offerta sacra, anátema (in latino devotio, nel senso di votare alla divinità cose, persone, città, e quindi non utilizzarle più per gli usuali scopi), alle origini del cristianesimo, il corrispondente latino rappresenta una forma di condanna ecclesiastica e comunitaria, religiosa e sociale insieme, messa in atto dalle comunità cristiane, che la hanno ereditata in tal senso dal giudaismo. Dalla fine del VI secolo, anatema si è distinto dalla scomunica. Ed ha significato più specificamente una esclusione grave, rivolta contro eretici e scismatici. Da allora, più severo della scomunica, esso consiste in una separazione dalla Chiesa, e ha implicato nel tempo anche preghiere di maledizione. La scomunica (excommunicatio,

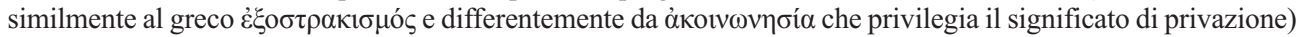
invece, ha significato allontanamento dai sacramenti e dalla partecipazione alla liturgia. (cfr. A. Blaise, Dictionnaire latin-français des auteurs chrétiens, Turnhout, 1954, p. 325). La scomunica, inoltre, legata il più delle volte alla prassi penitenziale, non coincide, però, del tutto con la penitenza e, comunque, consente il rientro del peccatore. Tra l'una e l'altra, oltre che la vita comunitaria si gioca la vita ultraterrena ( $c f r$. J. Gaudemet, «Note sur les formes anciennnes de l'excommunication», Rev. Sc. Rel. 23, (1949), pp. 64-77; V. Grossi, s.v. Anatema, in NDPAC, I, coll. 279-280 e Scomunica, in NDPAC, III, coll. 4788-4789 e A. Vacant, s.v. Anathème, DThC 1,1, coll. 11681171 e E. Valton, s.v. Excommunication, DThC 5, 1, Paris, 1924, coll. 1734-1744). Nel nostro periodo sono ancora usati indifferentemente come condanna ed esclusione dalla comunità. Anatema oggi è caduto in disuso.

${ }^{51}$ Il concetto di fuori uscire dai confini della comunità è soprattutto in lingua latina, in greco prevale la condizione di assenza ( $\dot{\alpha} \kappa o l v \omega v \eta \sigma i ́ \alpha)$. 
mantenere il colpevole nel corpo vivo della società. E, in questa chiave di comunicazione del potere, la scomunica è un po' come una morte di significato spirituale e un po' come una metafora dell'esilio.

Normalmente ricondotta in termini cristiani al detto di Gesù sul legare e sciogliere $^{52}$, e, comunque, assumendo la funzione di permettere un percorso di reintegrazione, la scomunica è, come primo dato, l'allontanamento dalla comunità. E, in quanto allontanamento dalla comunità, era una sanzione prevista già dall'AT. A esso si riferiva, sia pur in forma non tecnicamente rilevante sul piano normativo quanto piuttosto come sollecitazione morale, anche l'apostolo Paolo, che parla di allontanamento del colpevole e della necessità di interrompere i rapporti con lui ${ }^{53}$.

Le lontane origini di una connotazione sacrale, che tende ad eliminare il colpevole in quanto elemento impuro, restano, a nostro parere, ben evidenti in uno degli effetti essenziali della scomunica. Sulla linea del dettato paolino, infatti, si impone ai membri della comunità di non restare in comunione con un peccatore. E chiunque avesse mantenuto contatti con un condannato avrebbe a sua volta subito la scomunica: una sorta di effetto transitivo e fisicamente contaminante della colpa, dunque, sposta la questione dal tema della colpa morale a quello antropologico della colpa come impurità fisica.

Certo, la scomunica ha alla sua origine un valore etico e si connota soprattutto e sostanzialmente secondo una valenza morale e religiosa di purificazione. Ma, essa si concretizza in una dimensione fisica e corporale di esclusione dello scomunicato dalla partecipazione alla vita comunitaria. Nell'un caso e nell'altro, la linea del confine, che nell'esilio è tracciata a delimitare territori geografici -la famiglia la casa la patria da un lato e le zone dell'esilio dall'altro-, nella scomunica traccia invece un solco tra la comunità e i suoi spazi sacri da un lato e il corpo fisico e la persona del peccatore dall'altro.

La comunione e l'allontanamento dalla comunione sono le coordinate fondamentali della realtà religiosa cristiana, in quanto hanno significato teologico, liturgico-sacramentale e socio-religioso. Ognuno di questi riferimenti ha al centro un corpo: questo è nella simbologia cristica del corpo mistico di Cristo, nella metafora dell'unità del corpo della Chiesa e della comunità dei cristiani, è la realtà fisica di ciascun fedele attraverso il cui corpo passano salvezza e dannazione. Infatti, il destino di salvezza di un cristiano è visibilmente e pubblicamente segnato dai rapporti anche concreti e materiali tra la sua persona fisica -il suo corpo-e la comunione fisica e religiosa con il clero e con la comunità dei fedeli -il corpo della Chiesa-. Il dato sociale del suo allontanamento fisico dalla comunione dei fedeli è più che un dato esteriore e pubblico: è il segno della sua condizione spirituale di salvezza o di condanna, contrassegnato anche dai confini del corpo della Chiesa entro i quali o oltre i quali il fedele si colloca fisicamente e spiritualmente, avendo o meno accesso ai suoi luoghi e ai suoi riti.

La totale messa al bando, sia pur limitata nel tempo, da contatti fisici, morali e liturgici, quale è la scomunica, è una forma estrema -pur nelle sue variabili temporali che vanno da pochi o molti anni fino in extremis- rispetto ad altre forme punitive

\footnotetext{
${ }^{52}$ Mt 18, 15-17 e 16, 19 e 18, 18 (cfr. Giov 20, 23).

532 Tess. 3, 14; 1 Cor 5, 4-5 e 7, 13; 2 Cor 2, 5-11; 1 Tim. 1, 19.
} 
di minore gravità, ma dove il codice di comunicazione è dato egualmente dall'esclusione fisica alla partecipazione comunitaria, che rappresenta fisicamente l'esclusione dalla salvezza, e che è contrassegnata dal collocare al contempo il peccatore entro specifici gruppi, al fine di un percorso progressivo di redenzione: i peccatori sono puniti, così, con l'essere posti, secondo tempi stabiliti, prima tra gli uditori, poi tra i penitenti, successivamente in un gruppo contraddistinto dal fatto di avere consentita una partecipazione limitata alla comunione, solo nella preghiera e senza l'offerta, prima che $\mathrm{i}$ componenti potessero essere reintegrati ${ }^{54}$.

Le norme ecclesiastiche costruiscono, così, un sistema punitivo di ingabbiamento per gruppi o classi, talvolta serrando il confine che separa ciascun gruppo dagli altri e rendendo complessa la mobilità da un gruppo all'altro, in altri casi aprendo con elastica mobilità segmenti di slittamento e di comunicazione tra gruppi. A partire dalla scomunica -e posto che questa non sia fissata in extremis - si costruisce così un percorso di graduale reintegrazione, attraverso gruppi che funzionano come gradi ascensionali di una progressiva purificazione fino alla riammissione finale alla comunione dei fedeli nella partecipazione liturgica ${ }^{55}$.

Il confine, in quanto segno che delimita uno spazio fisico o astratto, anche nella normativa cristiana definisce la condizione di essere dentro o essere fuori un dato gruppo religioso. Ciò significa riconoscimento e consenso nel primo caso o emarginazione e censura nel secondo.

Significative sono, in tal senso, le punizioni riservate ai membri del clero, sempre nel segno dell'esclusione fisica e partecipativa, stabilita in varie forme fino a stabilizzarsi, nella gran parte dei documenti normativi del IV secolo -concili e decretali-, nella deposizione soltanto. Non è prevista, invece, la scomunica.

Nei primi concili, la deposizione non è ancora fissata come forma quasi esclusiva di sanzione per il clero. Così, per esempio, ad Ancira ${ }^{56}$, i presbiteri che, sottoposti a supplizi, e che abbiano subito violenza con evidenza ed escludendo qualunque mistificazione, hanno sacrificato e poi si sono pentiti, possono mantenere il loro ruolo, ma restano esclusi dal celebrare, predicare o esercitare altra funzione sacerdotale. Ancora a Neocesarea $^{57}$, anche un membro del clero poteva essere colpito da scomunica, in base alla gravità del peccato.

Nella seconda metà del IV secolo, invece, un ecclesiastico è punito quasi esclusivamente con la deposizione. Sembra potersi dedurre che, con il rafforzamento della gerarchia e l'irrigidimento delle classi socio-religiose, la perdita della carica fosse in sé elemento sufficiente per punire un membro del clero di qualunque colpa: non era importante calibrare la gravità del peccato bastando per tutti i membri del clero e per qualunque colpa la sola esclusione -fisica, religiosa, sacramentale- dal gruppo di appartenenza.

Il sistema punitivo cristiano antico, fondato sulla scomunica come forma più grave di punizione, che è, come abbiamo visto, una condanna in chiave di esclusione fisico-

\footnotetext{
${ }^{54}$ Conc. Anc., cann. 4-5-6-16 e passim.

${ }^{55}$ Cfr. Conc. Anc., can. 1; Conc. Nic., cann. 16 e 18.

${ }^{56}$ Can. 1.

${ }^{57}$ Can. 1.
} 
sacramentale, sembra lasciare fuori del tutto la condanna a morte: questo almeno in campo di norme ecclesiastiche e in linea di principio come sistema generalizzato.

In realtà, come abbiamo già visto, se la scomunica è apparentata all'esilio, e quest'ultimo, nell'ambito del sistema repressivo penale antico, non è solo un sostitutivo, ma di fatto è anche un surrogato della condanna a morte, anche la scomunica è, in realtà, una forma di condanna a morte.

Lo sarà certamente più tardi e nella considerazione di una società cristiana occupata da una Chiesa forte, giuridicamente attrezzata e che nel tempo imparerà a perfezionare l'uso della scomunica piegandola sempre più a strumento di terrore e tralasciandone la fruizione come percorso di recupero del fedele, come era nell'antichità ${ }^{58}$. Nei testi normativi dei primi secoli, infatti, l'attenzione al percorso di redenzione e alla reintegrazione del peccatore ha generalmente la meglio sulla volontà punitiva fine a se stessa. La situazione cambierà nel tempo fino all'età della Inquisizione, quando, anche se il colpevole si pentirà e sarà perdonato, non solo vi sarà la condanna a morte, ma questa verrà anche eseguita lo stesso ${ }^{59}$. La scomunica, dunque, -in quanto esclusione dalla vita comunitaria che dà la salvezza, cioè la vera vita- sostituisce sì la condanna a morte veterotestamentaria, ma alla morte è anche associabile: è un paragone che troviamo esplicitamente indicato più avanti nel tempo, nella prima decretale -la $\mathrm{Ad}$ Gallos, del 383 e 384-, che ce ne rende con chiarezza il senso.

Qui, la vergine velata che si sposa con un uomo, in quanto già sponsa Christi, è considerata colpevole di adulterio, come il marito. E, in quanto adulteri, sia la sponsa Christi che il marito vengono scomunicati. Costoro «hanno agito contro il comando dell'Antico Testamento. La legge ordina che costoro vengano lapidati. Ma, ora, essendo cessate quelle prescrizioni vendicative, i peccatori devono essere puniti in modo spirituale: così che non possano entrare in Chiesa, come se fossero morti ... Conviene che siano sospese dalla comunione ... che compiano una degna penitenza, che, col pianto l'umiltà, il digiuno, gli atti di misericordia, si redimano dalla colpa commessa» ${ }^{60}$.

Il riferimento alla Legge è alle modalità della sua giustizia e allo spirito che la anima, spirito che è fondamentalmente vendicativo: come in tutte le società antiche, il mai perduto significato sacrale della condanna a morte vuole l'eliminazione del colpevole, sia per pareggiare i conti del danno subito sia per purificare, con il di lui sangue, il sangue da lui versato. Rispetto alla Legge, richiamata come prima fonte di giustizia, vi è la spiritualizzazione della condanna a morte e il cambiamento di senso, che da vendicativo, quale era nell'AT diventa redentivo nel cristianesimo. Ma, nel passaggio dalla eliminazione fisica assoluta della condanna a morte, alla scomunica, alla quale, in vista della reinte-

\footnotetext{
58 Questo percorso è, però, già avviato nelle decretali ( $c f r$. Sardella, Dalla scomunica, cit. sotto stampa).

${ }^{59}$ La spiegazione è che la morte fa parte del processo penitenziale e purificatorio. Da considerare, inoltre, che, sempre durante l'Inquisizione, verrà introdotto l'uso del carcere, che rappresenta il capostipite politicoideologico del penitenziario ottocentesco (cfr. E. Gallo, Il carcere in Europa. Trattamento e risocializzazione, recupero e annientamento, modelli pedagogici e architettonici nella galera europea, Verona, 1983, pp. 28-38).

${ }^{60}$ Ad Gallos 3, (A. Di Berardino (ed.), I canoni dei concili della Chiesa antica, II. I concili latini. 1. Decretali, concili romani e canoni di Serdica (eds. T. Sardella- C. Dell'Osso), Roma, 2008, trad. it., p. 39).
} 
grazione, si attribuisce il valore di morte spirituale, permane il significato purificatorio dell'allontanamento fisico, sia pure temporaneo, del peccatore dal corpo ecclesiastico.

Il paragone tra condanna a morte e scomunica suggerisce anche altre riflessioni. Il codice di comunicazione del potere in tutti questi casi -condanna a morte, esilio, scomunica- si avvale, come abbiamo detto, di una comune semeiotica relazionale di lontana ascendenza sacrale centrata sull'espulsione, sia pure temporanea, del corpo del colpevole dal corpo della società.

Ma, vi sono anche delle differenze. Si parte dall'ovvia considerazione che la differenza fondamentale tra la prima -la condanna a morte- e i secondi -esilio e scomunica- sta nella sopravvivenza del colpevole.

Questo dato va completato, però, con la precisazione di un diverso significato nell'uso della pena che solo nel cristianesimo è volta alla redenzione del colpevole. Evidentemente non si pone il problema del recupero del colpevole l'autorità che condanna a morte. Del resto, non se lo pone nemmeno l'autorità che condanna all'esilio che, secondo la definizione ciceroniana, non è né diritto né pena, ma rifugio ${ }^{61}$. In entrambi i casi, il potere mette in atto un messaggio fondato su una sanzione che, essendo soprattutto di difesa da un fatto e da un corpo estraneo ed inquinante, diventa vendicativa e punitiva $^{62}$.

Anche l'autore della decretale è consapevole che la condanna a morte dell'Antico Testamento è una vindicta. E proprio contro il meccanismo della vindicta fine a se stessa si oppone la decretale stabilendo la scomunica, che non vuole essere punizione fine a se stessa ed è volta al recupero e alla salvezza del colpevole ${ }^{63}$ : il periodo della scomunica va impiegato in forme stabilite di penitenza che comunichino il pentimento del peccatore.

In che modo e a quali condizioni?

La gran parte della normativa, in questo periodo, non offre chiarimenti. Si parla poco dell'articolazione dei periodi della penitenza e sembra che la permanenza al di fuori della comunità, in gruppi di preghiera a partecipazione liturgica diversamente limitata - di cui si è già detto- concluda la questione ${ }^{64}$. Pochissimo è detto per quanto riguarda la liturgia del potere nella penitenza: solo i più tardi Statuta ecclesiae anti-

\footnotetext{
${ }^{61}$ Così Cicerone scrive: Exilium non supplicium est, sed perfugium potusque supplicii, («L'esilio non è una pena, ma un rifugio e una via di scampo rispetto alle pene») (Pro A. Caecina oratio 34, 100, ed. A. Boulanger, Paris, 1929, p. 136). Rispetto al fatto che l'esilio non è diritto, né pena, ma rifugio, gli storici del diritto discutono tuttora se l'esilio - nella sua figura originaria, in Grecia e a Roma - debba essere considerato come l'esercizio di un diritto o come una situazione penale: $c f r$. F. Ricciardelli, Le modalità dell 'esclusione politica a Firenze nel tardo Medioevo, in Escludere per governare. L'esilio politico fra Medioevo e Risorgimento, a cura di F. Di Giannatale, Milano, 2011, pp. 32-48 (anche in Reti Medievali, www.biblioteca.retimedievali.it).

${ }^{62}$ Più complesso il valore di deterrenza che si sviluppò in connessione con la progressiva perdita del significato sacrale della pena di morte e dell'esilio e l'acquisizione del principio di difesa della società: $c f r$. B. Santalucia, «Osservazioni sulla repressione criminale romana in età regia», Le délit religieux dans la cité antique, Rome, 1981, p. 47; Id., «Dalla vendetta alla pena», Storia di Roma, I, Torino, 1988; A. Calore, «La 'pena' e la 'storia'», Diritto e storia 3 (2004).

${ }^{63}$ Siric., ep. Himerio 3, trad. cit., p. 67: «[...] non vogliamo la morte del peccatore, ma solo che si converta e viva».

${ }^{64}$ Conc. Anc., cann. 6-9.
} 
$q u a$ parlano dei gesti rituali -prima, alla fine e durante il percorso penitenziale ${ }^{65}$-. I testi più antichi non parlano dei rituali istituzionali, dei gesti dei vescovi che sanciscono l'esclusione o la recuperata comunione del penitente e dello scomunicato. Sono tutti concentrati sul comportamento del peccatore. Innanzi tutto, il comportamento serve a capire la reale portata della colpa e, conseguentemente, a stabilire la pena.

Ad Ancira, non è considerato colpevole il comportamento di coloro che, durante le persecuzioni, dopo essere stati costretti a compiere atti di culto, lo fecero «....continuando, comunque, a confessare di essere cristiani, e che mostrano sempre il dolore di quanto è loro accaduto con ogni modestia, comportamento e umiltà di vita, a costoro non si proibisca la comunione, dal momento che non hanno commesso peccato» ${ }^{66}$.

Il comportamento durante la persecuzione è considerato strumento di conoscenza per capire il significato di un'azione colpevole, che sia stata operata con costrizione. Un certo comportamento, dissociando il fedele dall' atto criminoso da lui stesso compiuto, gli evita la scomunica.

Mangiare ai banchetti, o in luoghi destinati a pagani, cibo proprio, non proveniente da sacrifici, è colpa considerata con indulgenza ${ }^{67}$.

Allo stesso modo, è sempre il comportamento tenuto durante la penitenza imposta che, dimostrando autentico pentimento, consente di ottenere il perdono ${ }^{68}$.

La penitenza, attraverso i gesti del corpo e seguendo procedure di comunicazione fissate nei tempi e nei modi, deve rappresentare visibilmente una condizione dell'anima, quella del suo pentimento.

Il punto è che questo avviene in modo da offuscare i confini della coscienza: atteggiamenti di spontaneo pentimento e atteggiamenti imposti dall'autorità ecclesiastica si confondono e si sovrappongono. E impongono alcune riflessioni sui rapporti tra il linguaggio del corpo e il potere.

Strumento attraverso il quale si è evidenziata la colpa, il corpo, attraverso l'automortificazione imposta ed espressa negli atti penitenziali, comunica il pentimento e si apre alla possibilità del perdono.

I trattati, a proposito del pentimento, fanno di lamenti, lacrime e digiuni uno strumento di comunicazione, non piegato a formule normativizzate, quantificate nei tempi e nei modi ${ }^{69}$. Le modalità della penitenza, ivi suggerite, avvicinano quest'ul-

\footnotetext{
${ }^{65}$ Cfr. cann. 20-21-66-67, (ed. A. Di Berardino (ed.), I canoni dei concili della Chiesa antica, II. I concili latini. 2. I concili gallici, I (eds. R. Barcellona-M. Spinelli), Roma, 2010, pp. 99-105).

${ }^{66}$ Conc. Anc., can. 3, trad. it., p. 271.

${ }^{67}$ Conc. Anc., can. 7.

${ }^{68}$ Conc. Anc., can. 16: «Sia tenuta sotto osservazione la loro vita durante la penitenza e così ottengano il perdono». Conc. Neoc., can. 3, parla di buona condotta e fede.

${ }^{69}$ Lo stesso significato etico, e non giuridico, ha l'esortazione scritturistica che è promessa di salvezza (IS 30, 15). In Ambrogio, il diavolo lamenta che «Cristo non subisce danno quando persone venute da me [...] ritornano nel seno della Chiesa. Potrebbero imparare che non c'è vantaggio a stare in un luogo in cui gli uomini non sono affatto adescati dai beni immediati e che, invece, c'è profitto grandissimo dove i lamenti, le lacrime, i digiuni sono preferiti alle opulente imbandigioni» (De poenit. 1, 5, Roma, 1996, trad. it., p. 47). E, ancora, «E' un indizio di temeraria tracotanza, e non certo di santo timore, il fatto che odiate le persone che vogliono fare penitenza. Si sa, non ve la sentite di sopportare piagnistei di gente in lacrime. I vostri occhi non tollerano vesti miserande, squallore di persone in gramaglie» (ibid., 1, 8, 37, trad. cit., p. 52 s.).
} 
tima e allo stesso tempo la distinguono dalle sanzioni previste dal diritto ${ }^{70}$, ma anche dal successivo uso del monastero come carcere ${ }^{71}$.

Sia la penitenza che la reclusione in monastero come punizione tendono alla purificazione, ma anche al ravvedimento e al recupero del peccatore. Ma, la penitenza antica è contraddistinta anche dalla pubblicità dell'esperienza e dall'imposizione di specifici comportamenti ${ }^{72}$. Questi anticipano da un lato un concetto di sofferenza fisica educativa ${ }^{73}$ e dall'altro fanno del corpo la via di accesso per guardare dentro coscienza e volontà di fronte al peccato: il corpo è, così, strumento di comunicazione per valutare le pregiudiziali all'azione, ma anche la volontà che la ha sostenuta, e, in base a queste, giudicare e punire.

Rispetto ad altri rituali che rappresentano la traduzione pratica e materiale di mito, dottrina e teologia, la penitenza è meno ingabbiata in forme vincolanti, è tendenzialmente più aperta a comportamenti più individualizzati e più liberi rispetto al rigido protocollo spazio-temporale del calendario liturgico e delle sue prescrizioni. Talvolta, essa partecipa della durezza delle sanzioni fisiche e rientra, così, nel significato delle sanzioni all'interno del sistema di comunicazione legato al corpo e al potere, dove spazio significativo hanno le punizioni corporali, legate alla sofferenza procurata e al supplizio.

Nella volontà di fare e vedere soffrire il peccatore perché, attraverso la sofferenza, questi espii il male da lui stesso compiuto, il potere ecclesiastico si adegua agli orientamenti di quelle forme di potere che impongono una sofferenza, che, però, in questo caso, non è supplizio. Questo concetto conduce alla comprensione dei retroscena psicologici, comuni e uniformi, in questo caso a altri tipi di repressione penale:

${ }^{70}$ Il rapporto tra diritto e sanzione -nei vari significati- è tema fondamentale degli studi giuridici, che se ne occupano fin dalle origini della sua applicazione ( $c f r$. i classici G. Grosso, Problemi generali del diritto attraverso il diritto romano, Torino, 1967, pp. 17 ss.; E. Cantarella, Norma e sanzione in Omero: contributo alla protostoria del diritto greco, Milano, 1979, pp. 295 ss.. Più di recente, fondamentale è M. Foucault, op. cit., 1993; V. Ferrari, «Norme e sanzioni sociali», Enciclopedia delle scienze sociali, Roma, 1996; P. Erdö, Storia della scienza del diritto canonico: una introduzione, Roma, 1999; Cfr., inoltre, P. Biondi, Studi sul potere, Soveria Mannelli (Cz), 2004, soprattutto pp. 99 ss.; F. Cordero, Gli osservanti. Fenomenologia delle norme, Torino, 2008; C. Peloso, Studi sul furto nell'antichità mediterranea, Padova, 2008. Cfr., inoltre, gli Atti del Convegno dell'ARC, Organizzare Sorvegliare Punire. Il controllo dei corpi e delle menti nel diritto della tarda antichità. SpelloPerugia, 25-27 giugno 2009), sotto stampa.

${ }^{71}$ L'uso penale del carcere, escogitato dalla Chiesa già nel IV secolo, fu assicurato con la reclusione in monastero e applicato nei confronti dei religiosi come disposizione disciplinare: $c f r$. S. Pricoco, «Il cenobio come rifugio e come prigione», Studi in onore di F.E. Sciuto, Siculorum Gymnasium 49 (1996) pp. 225-237; V. Panzani, «La Chiesa e il carcere», soprattutto la parte riguardante «Le origini del carcere ecclesiastico», L'altro diritto. Centro di documentazione su carcere, devianza e marginalità (http://www.altrodiritto.unifi.it).

${ }^{72}$ Gli studi sulla penitenza hanno anche notato, ma senza ulteriori approfondimenti, la esteriorità dei segni di pentimento richiesti ( $c f r$. Vogel, op. cit., p. 20: «Cette attitude intérieure doit se traduire au-dehors, non par un aveu public au sens d'une confession détaillée devant la communauté [...]»; Panzani, op. cit., ha notato che prima viene la punizione per ravvedimento e, dopo l'alleanza con potere civile e nel modificato rapporto con il potere, quella retributiva, con la pubblicità della pena).

${ }^{73}$ C. Manunza, (L'Apocalisse come 'actio liturgica' cristiana. Studio esegetico-teologico di Ap 1, 9-16; 3, 14-22; 13, 9-10;19, 1-8, Roma, 2012) riprende elementi della cultura greco-romana che, nonostante il dualismo corpo-anima, riconosceva al corpo un valore educativo, sofferenza compresa. Nel cristianesimo il tema è ripreso come necessità di una correzione attraverso le pene corporali (pp. 251 ss.). 
l'uomo, infatti, innanzi al compito di darsi una memoria, intesa come ricordo e propensione ad adempiere determinate condotte, ricorre al dolore, quale «coadiuvante più potente della mnemonica» ${ }^{74}$. La punizione, o meglio il supplizio pubblico, che nelle sue manifestazioni barocche raggiunge uno splendore senza precedenti, deve provocare negli spettatori aborrimento per l'atto vietato, con l'inevitabile effetto inibitorio nei confronti dell'atto stesso. Si impone così all'uomo, sensibile al dolore, il timore di essere punito e l'esecrazione del gruppo per il compimento dell'atto vietato, qualcosa che possa orientare i comportamenti in una direzione, voluta dalla collettività e, nella maggior parte dei casi, opposta a quella dei suoi istinti, nei confronti dei quali la legge esercita un effetto repressivo ${ }^{75}$.

In alcuni casi, come a Ancira, il linguaggio del corpo indica il grado di pentimento in rapporto all'atteggiamento tenuto ${ }^{76}$ : postura del capo, modo di parlare, o meglio il tacere, ma anche abbigliamento e capigliatura, un complessivo messaggio di umiltà che richiami modestia e obbedienza sono segni del corpo che comunicano uno stato della coscienza.

$\mathrm{A} \mathrm{Nicea}^{77}$, per tornare in seno alla Chiesa, vengono stabiliti condizioni e tempi necessari a quanti, volendo tornarvi, devono anche dimostrare con messaggi del corpo disposizione interiore e conversione. Perché realtà e verità dell'anima passano attraverso il corpo. Ed è da quanto può comunicare con il corpo che il peccatore può essere giudicato.

Già nei primi concili, si richiedono manifestazioni di dolore per il peccato e per la colpa commessa, si vuole sentire e vedere il pianto come segno di ravvedimento.

Il pianto del penitente, dunque, sembra indispensabile per la riammissione alla comunione. Non sarebbe un problema se questo fosse una spontanea manifestazione di dolore. Il punto è che questo pianto è esplicitamente richiesto, o direi piuttosto imposto, in quanto è condizione per ottenere il perdono. La sovrapposizione rispetto alla spontanea manifestazione di dolore è evidente, l'invasione nei territori della coscienza un fatto, la confusione tra forme di pentimento spontanee e imposte un dato.

Dalla necessità del pentimento autentico, la normativa del IV secolo scivola, così, verso una richiesta delle manifestazioni di tale pentimento che piega quest'ultimo verso un rituale fissato, un protocollo comunicativo che ne fa un linguaggio retorico del corpo.

Ancora molto attento alla sincerità del pentimento e alla spontaneità delle sue manifestazioni è il concilio di Nicea, che richiede «prova di autentico pentimento» ${ }^{78}$. Ma, alla vergine di Cristo che si sia sposata, la decretale Ad Gallos impone, per ottenere il perdono di sì grande misfatto, che ella «deve piangere moltissimi anni, perché ottenuto il frutto di una degna penitenza, possa alla fine giungere al perdono, sempre

\footnotetext{
${ }^{74}$ F. Nietzsche, Genealogia della morale. Opere, trad. it., VI, t. I, Milano, 1968, p. 259; S. Natoli, La salvezza senza fede, Milano, 2007, p. 92.

75 F. Cordero, Gli osservanti, Milano, 1967.

${ }^{76}$ Conc. Anc., cann. 3-4.

77 Can. 12, trad. cit. p. 25: «[...] quanti, infatti, tra di loro, col timore e le lacrime, la sottomissione e le opere buone, mostrano nei fatti, non nelle apparenze, la loro conversione, una volta trascorso il tempo stabilito [...] prenderanno parte alle preghiere, e il vescovo avrà allora la facoltà di riservare loro un trattamento più indulgente». Cfr. anche p. 19.

${ }^{78}$ Can. 11, trad. cit., p. 25.
} 
che, tuttavia, realmente pentitasi, compia opere di penitenza» ${ }^{79}$. Avvertiva il problema Innocenzo I, che raccomandava a Decenzio, nel 416, di stare attento alla confessione del penitente, al suo pianto e alle sue lacrime ${ }^{80}$. Ma, non è chiaro se Innocenzo si preoccupasse della sincerità delle manifestazioni o del fatto che il rito del pentimento si adempisse secondo quanto stabilito.

Piuttosto che il tema della coerenza e dell'adesione della coscienza al significato del rito sembra che l'attenzione si ponga verso le manifestazioni del dolore e il significato della sofferenza. In particolare, ci troviamo di fronte alla manifestazione di un'esperienza fisica istintiva -il pianto, le lacrime-, esperienza nella quale si convogliano problemi psico-fisiologici e storico-culturali, e che allo stesso tempo viene sottoposta al controllo del potere.

Le ricerche sul riso e sul pianto -quali manifestazioni umane istintive e, nelle loro testimonianze storiche, anche come problema culturale- certamente hanno dedicato minore attenzione al pianto rispetto al riso- ${ }^{81}$. E nessuno di questi studi mi sembra che si sia occupato di questa strana condizione nella quale l'istintività naturale del pianto si trova sul crinale che la divide dalla sua rappresentazione teatrale. Parafrasando Foucoault, ci potremmo chiedere da dove viene e che altro è questa strana e singolare richiesta del potere, di avere la prova di un dolore che si scioglia in pianto e che deve essere vissuto, e a lungo, e in una dimensione pubblica, se non una rappresentazione? In essa, la richiesta di insistere sulla forza di un'emozione, così prolungata nel tempo, comporta necessariamente il derubricarsi di ogni pur possibile iniziale stato emotivo verso una retorica espressione del linguaggio del corpo relativo al dolore e al pentimento.

In tal senso, la penitenza antica, che è penitenza pubblica e dunque necessariamente correlata a manifestazioni di un linguaggio corporeo e visibile, non può prescindere dal carattere della teatralità. E tanto più è regolamentata, impostata e conformata nei suoi riti e nelle apparenze dell'abito, della capigliatura e del portamento, tanto più pone problemi. Studi psicanalitici e psicologici si sono posti certo il problema del rapporto tra pianto spontaneo e pianto indotto -comprendendo in quest'ultimo sia quello dell'attore a teatro sia quello del paziente adagiato sul lettino dello psicanalista-. E ne hanno tratto conclusioni che ne evidenziano funzioni e modalità diverse -catartica, cognitiva, terapeutica- quasi sempre correlate a una qualche forma di comunicazione con il mondo. Dall'antropologia filosofica, poi, apprendiamo che, pur essendo, come il riso, manifestazione del corpo, a differenza del riso, «il pianto comporta una chiusura dell'uomo in se stesso, una esclusione del mondo dal proprio orizzonte e conseguentemente un isolamento dell'individuo» ${ }^{82}$.

\footnotetext{
${ }^{79}$ Ad Gallos 3, trad. cit., p. 39: cfr. T. Sardella, «Vincoli e divieti matrimoniali nelle prime decretali papali: peccato e reato nell'adulterio della sponsa Christi», Il matrimonio dei cristiani: esegesi biblica e diritto romano. XXXVII Incontro di studiosi dell 'antichità cristiana, Roma 8-10 maggio 2008, Roma, 2009, pp. 308-330; Ead., «Un percorso normativo in materia di controllo sessuale: 1'adulterio della sponsa Christi (Ad Gallos 3-4)», Organizzare Sorvegliare Punire ... op. cit., Atti, sotto stampa.

${ }^{80}$ Ed. cit., pp. 144 ss.

${ }^{81} \mathrm{Su}$ queste, cfr. T. Lutz, Storia delle lacrime. Aspetti naturali e culturali del pianto, Milano, 2002, p. 17.

${ }^{82}$ H. Plessner, Il riso e il pianto. Una ricerca sui limiti del comportamento umano, Milano, 2000, p. 18.
} 
In altri termini -psicanalisi, psicologia, antropologia filosofica- concordano sul fatto che il pianto esprima una manifestazione del corpo assolutamente istintiva e individuale, incoercibile e insopprimibile, dove entrano in gioco emozioni insondabili. Meno legato alla dimensione relazionale del riso, esso rappresenta il confine e il contatto tra il mondo e l'individuo entro il quale nessuno può entrare. E, dunque, lo storico, di fronte a testi normativi, che impongono un'esperienza emozionale dalla squisita dimensione e natura personali, si trova più che mai di fronte all'ennesimo problema di dare una spiegazione al complesso intreccio di relazioni tra l'individuo, il suo corpo e il potere.

Certamente lo storico sa anche che, per quanto altre scienze umane possano rinviare l'esperienza del pianto a manifestazioni psico-fisiche individuali, nulla è al di fuori della storia e della cultura, in quanto complesso di dati storicamente condizionati. E da Israele alla Grecia fino al sud Italia di qualche decennio fa ${ }^{83}$, tutta l'area del Mediterraneo conosce il carattere spettacolare del lamento di dolore individuale e del lamento funebre anche collettivo, dove è evidente che l'esperienza istintiva e personale del pianto è incanalata in un linguaggio del corpo corrispondente a una specifica forma di comunicazione. E, nella gran parte dei casi, struttura istituzionale e tecnica del lamento plasmano secondo un codice di comunicazione sociale, che coinvolge il corpo nei gesti, nelle parole e nei suoni verbali, l'esperienza di dolore del singolo individuo e del gruppo.

Il pianto di Achille e di Ecuba sono diversi dal lamento funebre delle donne lucane, che segue il protocollo di una presenza rituale, indefinita, monotona, con l'iterazione di modelli culturali di comportamento. Ma, in tutti questi casi, i codici normativi agiscono con la sottile induzione di modelli di comportamento sociale, non con il sistema coercitivo del diritto. E la eventuale sanzione sociale, per chi agisca al di fuori dei modelli di comportamento, si attiva a un livello diverso della sanzione giuridica. Nel cristianesimo, anche il dolore legato alla colpa commessa ha radici evangeliche nella teatralità dei comportamenti afflittivi del penitente ${ }^{84}$. Un caso eclatante, nel IV secolo, è quello di Teodosio, la cui richiesta di perdono passa attraverso forme di mortificazione spettacolari imposte da Ambrogio.

$\mathrm{Ma}$, a partire dai testi normativi del IV secolo, tutta la penitenza viene codificata attuando forme di quantificazione, che mettono in rapporto il tipo di colpa e l'espiazione, soprattutto imponendo un'esperienza corporea -quella del pianto- che per sua natura richiama manifestazioni fisio-psicologiche complesse. Forme e tempi imposti piegano inevitabilmente questa esperienza a tecnica di un linguaggio corporeo di forma certo nota, nelle esperienze del pianto rituale, ma di senso inedito. Soprattutto, il pianto imposto nella penitenza è un pianto apparentato con la retorica dei rituali. Il pianto di dolore è esperienza già antichissima nel cristianesimo antico. Ed esso, anche nelle forme di manifestazione spontanee o culturalmente indotte, ma non istituzionalmente imposte, rappresenta, comunque, uno dei tanti modi di comunicazione codificati socialmente e storicamente nei quali prende forma ogni sistema espressivo.

\footnotetext{
${ }^{83}$ E. De Martino, Morte e pianto rituale nel mondo antico: dal lamento pagano al pianto di Maria, Torino, 1958.

${ }^{84} \operatorname{Lc} 7,38$.
} 
$\mathrm{Ma}$, ordinare di piangere per dimostrare il pentimento è ben altra cosa: questo è orientare dall'alto un sistema di comunicazione. Il pianto imposto al penitente, codificato a livello normativo -e per di più a tempo e veicolato come strumento di salvezza- è un corto circuito nel sistema di comunicazione tra l'individuo e il potere. Le lacrime, in quanto esperienza individuale sono già «avvocati o oratrici, aiutano ad avere ragione» (Southwell), e funzionano, dunque, come naturale strumento corporeo di persuasione. In quanto costrizione e umiliazione sul corpo e attraverso il corpo -con i sistemi coercitivi che, peraltro, ogni potere conosce-falsano questo linguaggio corporeo, piegano la naturalità espressiva e di comunicazione di un'emozione, la ingabbiano e la trasformano, per così dire, in pura tecnica retorica al servizio del potere, ne fanno un nuovo strumento per piegare le coscienze. 